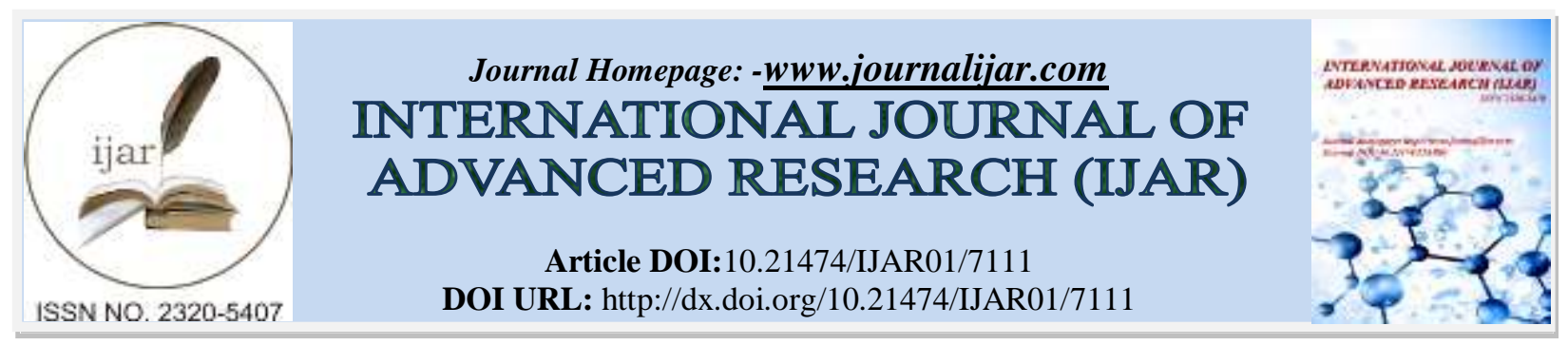

RESEARCH ARTICLE

\title{
"TO ASSESS KNOWLEDGE AND PRACTICE AMONG HEALTH CARE PROVIDERS REGARDING HOSPITAL WASTE MANAGEMENT IN SKIMS SOURA”.
}

\author{
Perkash Kour, Nazir Ahmad Dar and KS RAINA. \\ Sheri-Kashmir Institute Of Medical Sciences,Soura Srinagar Jammu And Kashmir.
}

\section{Manuscript Info}

Manuscript History

Received: 15 March 2018

Final Accepted: 17 April 2018

Published: May 2018

Keywords:-

Knowledge, Practice, Waste

Management, Doctors, Nurses, and

Technicians.

\section{Abstract}

Aim of the study: To Assess Knowledge and Practice among Health Care Providers Regarding Hospital Waste Management.

Conclusion: In our study, it was seen that nurses have more knowledge and practice regarding segregation than other studied subjects. Technicians were having more knowledge regarding legislation applicable to hospital waste management.

\section{Introduction:-}

Hospital is a place of almighty, a place to serve the patient. Since beginning the hospitals are known for treatment of sick persons, but we are unaware about the adverse effects of garbage and filth generated by them on human body and environment. Hospital waste is a potential health hazard to the health care workers, public and flora and fauna of the area. Hospital acquired infections, transfusions, transmitted diseases, rising incidence of Hepatitis\& HIV etc leads to increase in possibility of catching many diseases. This problem has now become a serious threat \& ultimately the central government had to intervene for enforcing proper handling \& disposal of hospital waste $\&$ an act was passed in July 1996 \& a biomedical waste (handling \& management) rule was introduced in 1998.

Hospital waste refers to all waste generated discarded and not intended for further use in hospital. India generates $1.5 \mathrm{~kg} / \mathrm{bed} /$ day hospital waste which includes both hazardous and non hazardous.

Hospital waste management collection and proper disposal has become a significant concern for both medical and general community.

Every health care personnel is expected to have proper knowledge, proper practice and capacity to guide others for waste collection and management and proper handling techniques

\section{Objectives Of The Study:-}

1. To assess the knowledge of health care providers regarding hospital waste management.

2. To find out existing practices of hospital waste management among selected sample.

3. To determine the association of knowledge \&Practice with selected factors:

1. Year of Experience.

2. Qualification.

3. Age.

4. Gender. 
5. Residence.

4. To assess the level of application of policies of the hospital by the health care providers.

\section{Methodology:-}

The research methodology refers to a set of orderly disciplined procedure involved in the purposeful collection analysis \&interpretation of the data. This chapter describes the research approach, research design, setting of study sample \& sampling technique, instrument for data collection \& plan for data analyzing of the present study.

\section{Research Approach:-}

Non-experimental based descriptive approach used for gaining pertinent \& precise information.

\section{Research Design:-}

A descriptive research design was considered appropriate for the present study to assess the knowledge \& practice among healthcare providers regarding hospital waste management in SKIMS, Soura Srinagar.

\section{Variables under Study:-}

The variables in the study to assess the knowledge \& practice of healthcare providers regarding hospital waste management are:-

1. Age

2. Sex

3. Residence

4. Educational Status (Academic)

5. Professional Education

6. Years of Experience

\section{Setting Of the Study:-}

The setting for the study was SKIMS Hospital, Soura for the following reasons:-

1. Sample was easily accessible

2. Less time consuming

3. Cost-effective

4. Cooperative staff

\section{Population:-}

The target population in this study consists of 50 healthcare providers of SKIMS from whom data was collected during the month of August.

\section{Sample \& Sampling Technique:-}

A non-probability sampling technique namely convenient sampling was used to make the study more practicable \& feasible. The selection of study sample was done based on population (Healthcare Providers) of SKIMS, who had agreed to participate in the study, 10 from each 5 selected areas (SICCU, Causality, General Medicine, General Surgery, and Neonatology Unit).

\section{Presentation And Analysis Of Data:-}

\section{Analysis Of Data:-}

The data was analysed according to the objectives of the study.Analysis of the data was done after the data was transferred to the master data sheet. The data was analysed based on the objectives and hypothesis.The data is presented in tabular and graphical form; percentages were calculated and interpreted as shown in tables and graphs, respectively

Table 1:-Frequency Distribution of Studied subjects with respect to knowledge about treatment of Hospital Waste.

\begin{tabular}{|c|c|c|c|c|c|c|c|c|c|}
\hline \multirow{2}{*}{$\begin{array}{c}\text { S. } \\
\text { No. }\end{array}$} & \multicolumn{2}{|c|}{ STATEMENT } & \multicolumn{2}{c|}{$\begin{array}{c}\text { DOCTORS } \\
\mathrm{N}=15\end{array}$} & \multicolumn{2}{|c|}{$\begin{array}{c}\text { NURSES } \\
\mathrm{N}=25\end{array}$} & \multicolumn{2}{c|}{$\begin{array}{c}\text { TECHNICIANS } \\
\mathrm{N}=10\end{array}$} & \multicolumn{2}{c|}{ TOTAL } \\
\cline { 3 - 9 } & & $\#$ & $\%$ & $\#$ & $\%$ & $\#$ & $\%$ & $\#$ & $\%$ \\
\hline 1. & $\begin{array}{l}\text { Biomedical waste management needs } \\
\text { special treatment than general waste. }\end{array}$ & 14 & 93.33 & 23 & 92 & 10 & 100 & 47 & 94 \\
\hline
\end{tabular}




\begin{tabular}{|c|l|c|c|c|c|c|c|c|c|}
\hline 2. & $\begin{array}{l}\text { Discarded medicine, psychotoxic drugs } \\
\text { undergo incineration type of treatment. }\end{array}$ & 6 & 4 & 16 & 64 & 2 & 20 & 24 & 48 \\
\hline 3. & $\begin{array}{l}\text { Black colour waste bins undergo municipal } \\
\text { disposal type of treatment. }\end{array}$ & 9 & 60 & 23 & 92 & 7 & 70 & 39 & 78 \\
\hline 4. & $\begin{array}{l}\text { Red colour bins undergo autoclaving type of } \\
\text { treatment. }\end{array}$ & 8 & 53.3 & 18 & 72 & 7 & 70 & 33 & 66 \\
\hline 5. & Final disposal of segregated waste. & 6 & 40 & 10 & 40 & 3 & 30 & 19 & 38 \\
\hline 6. & Broken thermometer waste is incinerated. & 12 & 80 & 19 & 76 & 5 & 50 & 36 & 72 \\
\hline 7. & $\begin{array}{l}\text { Incineration ash disposed in municipal } \\
\text { landfills. }\end{array}$ & 11 & 73.33 & 14 & 56 & 7 & 70 & 32 & 64 \\
\hline 8. & Inertization process. & 4 & 26.66 & 5 & 20 & 1 & 10 & 10 & 20 \\
\hline
\end{tabular}

Table 2:-Frequency Distribution of the knowledge of the studied subject about the irresponsibility of Health workers towards $\mathrm{H}$ W M.

\begin{tabular}{|c|c|c|c|c|c|c|c|c|c|}
\hline \multirow[t]{2}{*}{$\begin{array}{l}\text { S. } \\
\text { No. }\end{array}$} & \multirow[t]{2}{*}{ STATEMENT } & \multicolumn{2}{|c|}{$\begin{array}{c}\text { DOCTORS } \\
\mathrm{N}=15\end{array}$} & \multicolumn{2}{|c|}{$\begin{array}{c}\text { NURSES } \\
\mathrm{N}=25\end{array}$} & \multicolumn{2}{|c|}{$\begin{array}{c}\text { TECHNICIANS } \\
\mathrm{N}=10\end{array}$} & \multicolumn{2}{|c|}{$\begin{array}{c}\text { TOTAL } \\
\mathrm{N}=50\end{array}$} \\
\hline & & \# & $\%$ & $\#$ & $\%$ & \# & $\%$ & \# & $\%$ \\
\hline 1. & $\begin{array}{l}\text { Waste management is the responsibility } \\
\text { of Head of hospital }\end{array}$ & 11 & 73.33 & 20 & 80 & 7 & 70 & 48 & 96 \\
\hline & Head of department & 7 & 46.66 & 17 & 68 & 7 & 70 & 31 & 62 \\
\hline & Nursing supervisor & 11 & 73.33 & 21 & 84 & 8 & 80 & 40 & 80 \\
\hline & Hospital engineer & 5 & 33.33 & 04 & 16 & 3 & 30 & 12 & 24 \\
\hline & Infection control officer & 12 & 80 & 25 & 100 & 9 & 90 & 46 & 92 \\
\hline & Pharmacist & 1 & 6.66 & 6 & 24 & 3 & 30 & 10 & 20 \\
\hline & Lab supervisor & 6 & 40 & 18 & 72 & 5 & 50 & 29 & 58 \\
\hline
\end{tabular}

Table 3:-Frequency Distribution of Studied subject to practice with respect to regarding Handling of Hospital Waste.

\begin{tabular}{|l|l|l|l|l|l|l|l|l|l|}
\hline S. & STATEMENT & \multicolumn{2}{l|}{$\begin{array}{l}\text { DOCTORS } \\
\text { No. }\end{array}$} & \multicolumn{2}{l|}{$\begin{array}{l}\text { NURSES } \\
\text { N=25 }\end{array}$} & \multicolumn{2}{l|}{$\begin{array}{l}\text { TECHNICIANS } \\
\text { N=10 }\end{array}$} & \multicolumn{2}{l|}{ TOTAL } \\
\cline { 3 - 9 } & & $\#$ & $\%$ & $\#$ & $\%$ & $\#$ & $\%$ & $\#$ & $\%$ \\
\hline 1. & $\begin{array}{l}\text { Clearly defined procedures for collection } \\
\text { and handling of wastes from specified units } \\
\text { in hospital }\end{array}$ & 12 & 80 & 21 & 84 & 8 & 80 & 41 & 82 \\
\hline 2. & $\begin{array}{l}\text { Waste handler using any protective } \\
\text { clothing(gloves,etc.) }\end{array}$ & 15 & 100 & 22 & 88 & 9 & 90 & 46 & 92 \\
\hline 3. & $\begin{array}{l}\text { Collected waste is transported in a proper } \\
\text { way from the source }\end{array}$ & 11 & 73.33 & 20 & 80 & 4 & 40 & 35 & 70 \\
\hline 4. & $\begin{array}{l}\text { Segregated waste is properly stored,before } \\
\text { it is removed from the hospital }\end{array}$ & 7 & 46.66 & 22 & 88 & 6 & 60 & 35 & 70 \\
\hline
\end{tabular}

Table 4:-Frequency Distribution of Studied subject with respect to practice regarding training of Health personals towards Hospital Waste Management.

\begin{tabular}{|c|c|c|c|c|c|c|c|c|c|}
\hline \multirow[t]{2}{*}{$\begin{array}{l}\text { S. } \\
\text { No. }\end{array}$} & \multirow[t]{2}{*}{ STATEMENT } & \multicolumn{2}{|c|}{$\begin{array}{l}\text { DOCTORS } \\
\quad \mathrm{N}=15 \\
\end{array}$} & \multicolumn{2}{|c|}{$\begin{array}{c}\text { NURSES } \\
\mathrm{N}=25\end{array}$} & \multicolumn{2}{|c|}{$\begin{array}{l}\text { TECHNICIANS } \\
\mathrm{N}=10\end{array}$} & \multicolumn{2}{|c|}{$\begin{array}{c}\text { TOTAL } \\
\mathrm{N}=50\end{array}$} \\
\hline & & $\#$ & $\%$ & $\#$ & $\%$ & $\#$ & $\%$ & $\#$ & $\%$ \\
\hline 1 & $\begin{array}{l}\text { Ever attended seminar or workshop,etc. } \\
\text { regarding waste management }\end{array}$ & 4 & 26.66 & 13 & 52 & 4 & 40 & 21 & 42 \\
\hline
\end{tabular}

Table 5:-Frequency Distribution of Studied subject with respect to practice regarding implementation of right practice of Hospital Waste.

\begin{tabular}{|c|c|c|c|c|c|c|c|c|}
\hline \multirow[t]{2}{*}{$\begin{array}{l}\text { S. } \\
\text { No. }\end{array}$} & \multirow[t]{2}{*}{ STATEMENT } & \multicolumn{2}{|c|}{$\begin{array}{c}\text { DOCTORS } \\
\mathrm{N}=15\end{array}$} & \multicolumn{2}{|c|}{$\begin{array}{c}\text { NURSES } \\
\mathrm{N}=25\end{array}$} & \multicolumn{2}{|c|}{$\begin{array}{c}\text { TECHNICIAN } \\
\mathrm{N}=10\end{array}$} & $\begin{array}{c}\text { TOTAL } \\
\mathrm{N}=50\end{array}$ \\
\hline & & \# & $\%$ & \# & $\%$ & $\neq$ & $\%$ & \\
\hline
\end{tabular}




\begin{tabular}{|l|l|c|c|c|c|c|c|c|}
\hline 1. & $\begin{array}{l}\text { Consistency in the work of segregation of } \\
\text { wastes, even though there is heavy } \\
\text { workload in the area }\end{array}$ & 7 & 46.66 & 23 & 92 & 8 & 80 & 38 \\
\hline 2. & $\begin{array}{l}\text { Influence of effective practice of waste } \\
\text { management on patient care }\end{array}$ & 13 & 86 & 23 & 92 & 10 & 100 & 46 \\
\hline 3. & $\begin{array}{l}\text { Ever stopped a person performing wrong } \\
\text { practice of waste segregation in the } \\
\text { hospital }\end{array}$ & 9 & 60 & 22 & 88 & 8 & 80 & 39 \\
\hline 4. & $\begin{array}{l}\text { Implementation of practice of waste } \\
\text { management in real sense, according to } \\
\text { the policy of waste management }\end{array}$ & 8 & 53.33 & 22 & 88 & 10 & 100 & 40 \\
\hline
\end{tabular}

Table 6:-Frequency Distribution of Studied subject with respect to knowledge regarding segregation of Hospital Waste.

\begin{tabular}{|c|c|c|c|c|c|c|c|c|c|}
\hline \multirow[t]{2}{*}{$\begin{array}{l}\text { S. } \\
\text { No. }\end{array}$} & \multirow[t]{2}{*}{ STATEMENT } & \multicolumn{2}{|c|}{$\begin{array}{l}\text { DOCTORS } \\
\quad \mathrm{N}=15\end{array}$} & \multicolumn{2}{|c|}{$\begin{array}{l}\text { NURSES } \\
\mathrm{N}=25\end{array}$} & \multicolumn{2}{|c|}{$\begin{array}{l}\text { TECHNICIANS } \\
\qquad \mathrm{N}=10\end{array}$} & \multicolumn{2}{|c|}{$\begin{array}{c}\text { TOTAL } \\
\mathrm{N}=50\end{array}$} \\
\hline & & $\#$ & $\%$ & \# & $\%$ & $\#$ & $\%$ & \# & $\%$ \\
\hline 1. & $\begin{array}{l}\text { Awareness regarding segregation of } \\
\text { hospital waste }\end{array}$ & 14 & 93.33 & 24 & 96 & 10 & 100 & 48 & 96 \\
\hline 2. & $\begin{array}{l}\text { Segregation an important aspect of waste } \\
\text { management }\end{array}$ & 14 & 93.33 & 24 & 96 & 10 & 100 & 48 & 96 \\
\hline 3. & $\begin{array}{l}\text { Colour coded bins effective for hospital } \\
\text { waste management }\end{array}$ & 13 & 86.66 & 25 & 100 & 10 & 100 & 48 & 96 \\
\hline 4. & $\begin{array}{l}\text { Plastic containers are suitable for } \\
\text { segregation of hospital waste }\end{array}$ & 15 & 100 & 24 & 96 & 9 & 90 & 48 & 96 \\
\hline 5. & $\begin{array}{l}\text { Paper wastes, domestic wastes,etc., are } \\
\text { put in yellow colour bins }\end{array}$ & 9 & 60 & 18 & 72 & 6 & 60 & 33 & 66 \\
\hline 6. & $\begin{array}{l}\text { Human biopsy wastes, organs, } \\
\text { blood,pathological wastes are put in red } \\
\text { colour bins }\end{array}$ & 8 & 53.53 & 23 & 92 & 10 & 100 & 41 & 82 \\
\hline 7. & $\begin{array}{l}\text { Needles, cathters, angiocaths,etc., are put } \\
\text { in black colour bins }\end{array}$ & 8 & 53.53 & 23 & 92 & 7 & 70 & 38 & 76 \\
\hline 8. & $\begin{array}{l}\text { Is waste generated from emergency } \\
\text { wards more in amount than produced } \\
\text { from SICCU }\end{array}$ & 12 & 80 & 16 & 64 & 6 & 60 & 34 & 68 \\
\hline 9. & $\begin{array}{l}\text { Segregation is responsibility of every } \\
\text { health care provider }\end{array}$ & 15 & 100 & 24 & 96 & 10 & 100 & 49 & 98 \\
\hline
\end{tabular}

Table 7:-Frequency Distribution of Studied subject with respect to knowledge regarding availability of various facilities regarding hospital waste management.

\begin{tabular}{|c|c|c|c|c|c|c|c|c|c|}
\hline \multirow[t]{2}{*}{$\begin{array}{l}\text { S. } \\
\text { No. }\end{array}$} & \multirow[t]{2}{*}{ STATEMENT } & \multicolumn{2}{|c|}{$\begin{array}{l}\text { DOCTORS } \\
\mathrm{N}=15\end{array}$} & \multicolumn{2}{|c|}{$\begin{array}{l}\text { NURSES } \\
\mathrm{N}=25\end{array}$} & \multicolumn{2}{|c|}{$\begin{array}{l}\text { TECHNICIANS } \\
\mathrm{N}=10\end{array}$} & \multicolumn{2}{|c|}{$\begin{array}{l}\text { TOTAL } \\
\mathrm{N}=50\end{array}$} \\
\hline & & $\#$ & $\%$ & \# & $\%$ & \# & $\%$ & \# & $\%$ \\
\hline 1. & $\begin{array}{lcc}\text { Availability of manual } & \text { or } \\
\text { guidelines document } & \text { on } \\
\text { management of hospital waste } & \end{array}$ & 10 & 66.66 & 14 & 56 & 4 & 40 & 28 & 56 \\
\hline 2. & Management plan & 11 & 73.33 & 22 & 88 & 10 & 100 & 43 & 86 \\
\hline 3. & Management team & 10 & 66.66 & 23 & 92 & 9 & 90 & 42 & 84 \\
\hline 4. & $\begin{array}{l}\text { Waste gets reused or recycled in } \\
\text { the hospital }\end{array}$ & 2 & 13.33 & 6 & 24 & 1 & 10 & 9 & 18 \\
\hline
\end{tabular}


Table 8:-Frequency Distribution of Studied subject with respect to knowledge regarding awareness of legislation applied to hospital waste management.

\begin{tabular}{|l|l|l|l|l|l|l|l|l|l|}
\hline S. & STATEMENT & \multicolumn{2}{l|}{$\begin{array}{l}\text { DOCTORS } \\
\text { No. }\end{array}$} & \multicolumn{2}{l|}{$\begin{array}{l}\text { NURSES } \\
\text { N=15 }\end{array}$} & \multicolumn{2}{l|}{$\begin{array}{l}\text { TECHNICIANS } \\
\text { N=25 }\end{array}$} & \multicolumn{2}{l|}{ TOTAL } \\
\cline { 3 - 9 } & & $\#$ & $\%$ & $\#$ & $\%$ & $\#$ & $\%$ & N $=50$ \\
\hline 1 & $\begin{array}{l}\text { Awareness about any legislation applied } \\
\text { to hospital waste }\end{array}$ & 4 & 26.66 & 8 & 32 & 5 & 50 & 17 & 34 \\
\hline
\end{tabular}

Table 9:-Frequency Distribution of Studied subject with respect to knowledge regarding hazards of Hospital Waste Management.

\begin{tabular}{|c|c|c|c|c|c|c|c|c|c|}
\hline \multirow[t]{2}{*}{$\begin{array}{l}\text { S. } \\
\text { No. }\end{array}$} & \multirow[t]{2}{*}{ STATEMENT } & \multicolumn{2}{|c|}{$\begin{array}{l}\text { DOCTORS } \\
\mathrm{N}=15\end{array}$} & \multicolumn{2}{|c|}{$\begin{array}{l}\text { NURSES } \\
\mathrm{N}=25\end{array}$} & \multicolumn{2}{|c|}{$\begin{array}{l}\text { TECHNICIANS } \\
\mathrm{N}=10\end{array}$} & \multicolumn{2}{|c|}{$\begin{array}{l}\text { TOTAL } \\
\mathrm{N}=50\end{array}$} \\
\hline & & $\#$ & $\%$ & $\#$ & $\%$ & $\#$ & $\%$ & $\#$ & $\%$ \\
\hline 1. & $\begin{array}{l}\text { Biomedical waste is always } \\
\text { infectious. }\end{array}$ & 6 & 40 & 2 & 8 & 0 & 0 & 8 & 16 \\
\hline 2. & $\begin{array}{l}\text { Colour coding avoids mixing of } \\
\text { hazardous and non-hazardous } \\
\text { waste. }\end{array}$ & 15 & 100 & 24 & 96 & 9 & 90 & 48 & 96 \\
\hline 3. & $\begin{array}{l}\text { Improper } \\
\text { management is an important } \\
\text { cause of hospital acquired } \\
\text { infection. }\end{array}$ & 13 & 86.67 & 25 & 100 & 10 & 100 & 48 & 96 \\
\hline 4. & $\begin{array}{l}\text { Appropriate management of } \\
\text { hospital waste a critical } \\
\text { component of environment health } \\
\text { protection. }\end{array}$ & 15 & 100 & 23 & 92 & 10 & 100 & 48 & 96 \\
\hline
\end{tabular}

\section{Conclusion:-}

At the end of our study "A study to assess the knowledge and practice among the health care providers towards hospital waste management in SKIMS Soura."

Samples of 50 respondents, 15 doctors, 25 nurses, and 10 technicians were taken. Methodology used was "descriptive" and also used "questionnaire" and "structured interview schedule" as a tool for our study and collected data accordingly.

The data is presented in the form of graphs and tables, was collected through the questionnaire.

In our study, it was seen that nurses have more knowledge and practice regarding segregation than other studied subjects.

Technicians were having more knowledge regarding legislation applicable to hospital waste management. It was also evidentfrom the study that nurses were having more knowledge about treatment procedures available in SKIMS.

Financial support and sponsorship:- Nil

Conflict of interest:- There are no conflicts of interest. 
Distribution of studied subject on the basis of age groups:-

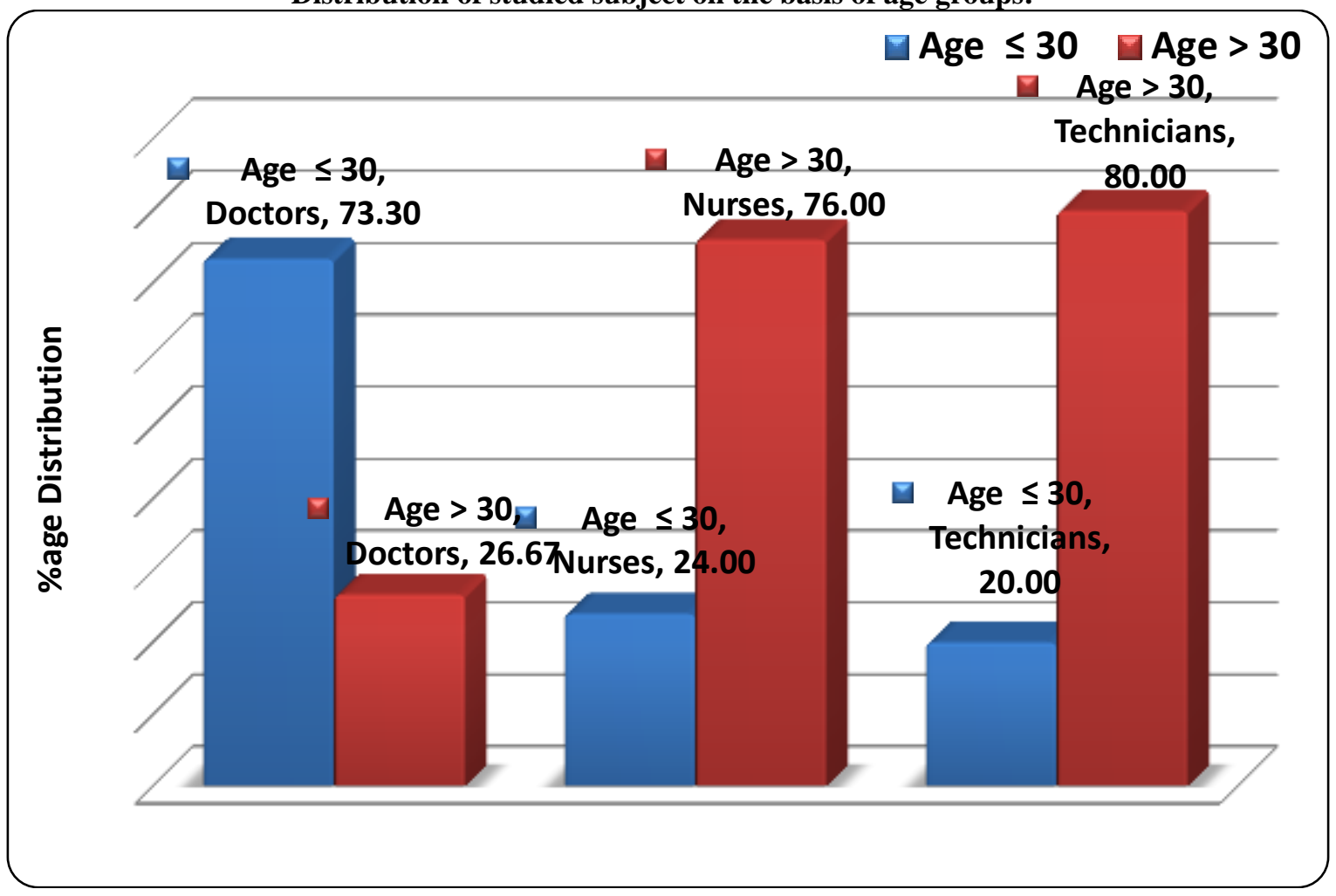

Interpretations:-

1. Among Doctors $73.3 \%$ are $\leq 30$ and 26.67 are $>30$ years of age.

2. Among Nurses $24 \%$ are $\leq 30$ and 76 are $>30$ years of age.

3. Among Technicians $20 \%$ are $\leq 30$ and $60 \%$ are $>30$ years of age.

Pie Chart showing distribution of Gender among the studied subjects.

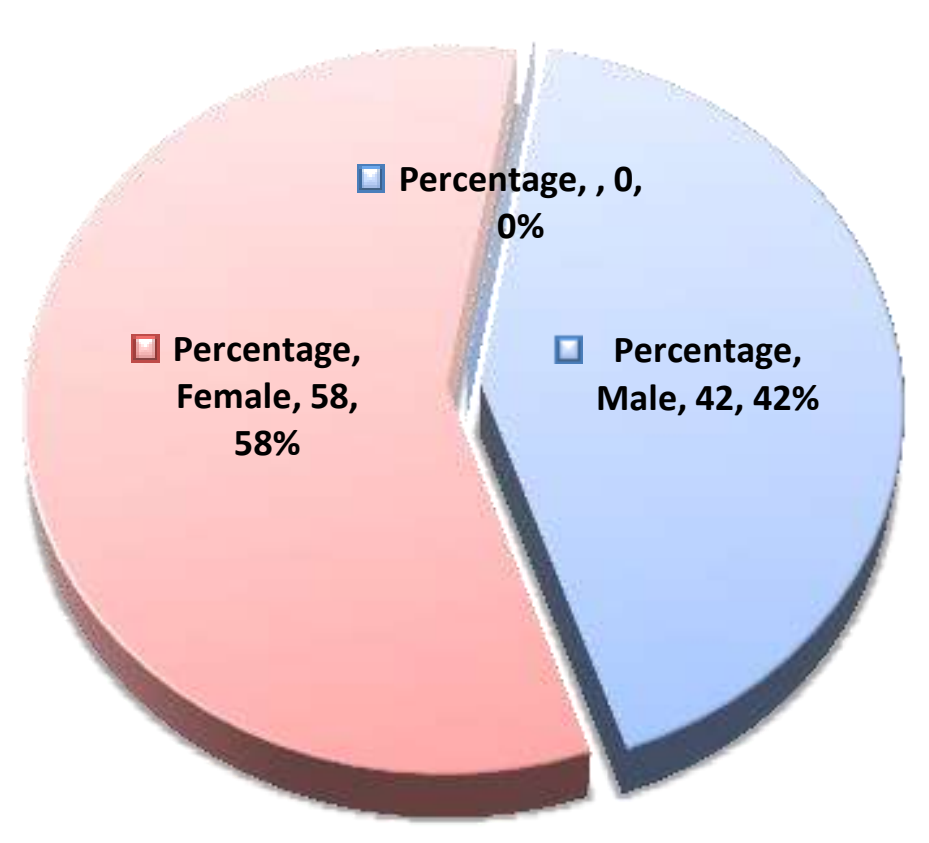




\section{Interpretations:-}

Among the studied subjects $42 \%$ are male and $58 \%$ are female, which include doctors, nurses and Technicians.

Pie Chart showing distribution of Residence among the studied subjects.

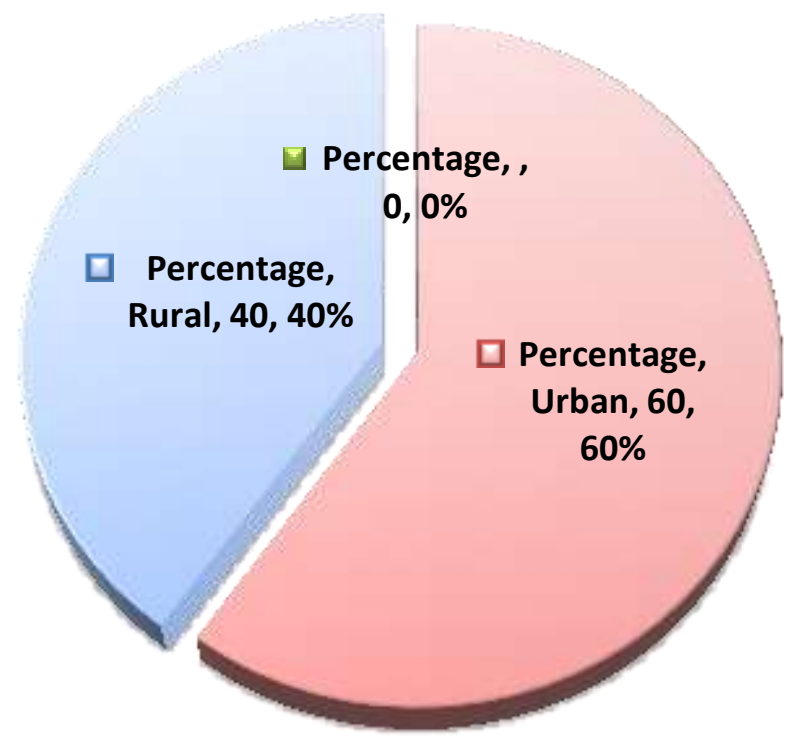

Interpretations:-

Among the studied subjects $60 \%$ belong to urban areas and $40 \%$ belong to rural areas, which include doctors, nurses and Technicians.

Pie Chart showing distribution of Professional Education of the studied subjects.

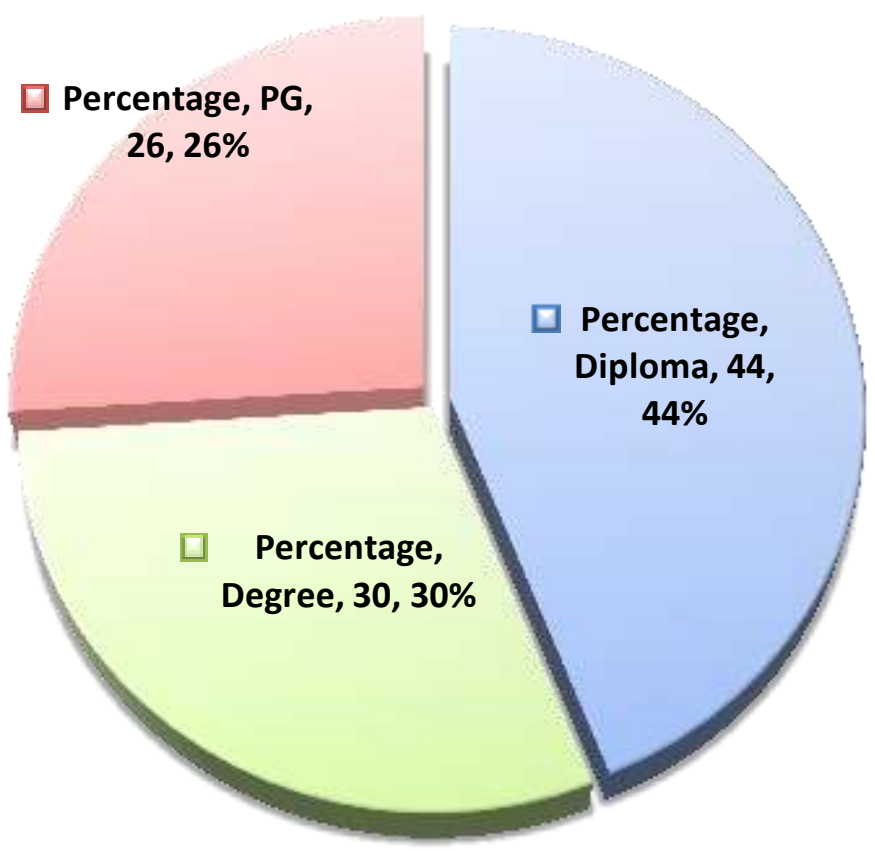




\section{Interpretations:-}

Among the studied subjects 44\% are Diploma Holders, 30\% are Degree Holders and 26\% are PG's which include doctors, nurses and Technicians.

\section{Bibliography:-}

1. LalitaArora and SunitaAgarwal (April 2011) international Journal of chemical, environmental and pharmaceutical research vol. 2,No 1,40-43.

2. Stewart Barr (2007),U K Study of Household waste Management.

3. RawshanAra Begum (17 March,2009).

4. Kulatunag\&Amuratunga (Year 2006).

5. Harcourt Hospital (2008), Port Harcourt Medical Journal.

6. Sir Syed College of Medical Sciences, Pakistan (2008), Journal of Sir Syed College of Medical Sciences.

7. TudestoRamokotaDebashisBasu(2006), American Medical Journal.

8. N. B. Pandith, H.K. Mehta, G.P. Kartha\& S.K Chourdary (2005), Indian Journal of Public Health.

9. M.C YadavannavarAditya S. Berad\& P.B Jagirdas(2007), Cross Sectional Study Bijapur.

10. Lalji K. Verma, Shyamalla Mani, NituSinha\&SunitaRana(2008) Volume 28 issue-12, Pages 2723-2734, Delhi.

11. Mostafa(2007), Cross sectional Study.

12. S.Saina, S.S Nagarayan(2005), Vol-17,No.2, KAP Study.

13. MohdShafee, N.B Kasturwar, N.N Rupama(2009), AP

14. Gaza Study, Vol-7, No.-6, Nov 2010, P.No.-10171024.

15. P.HanumanthaRao, Center for Human Development, Administrative Staff, College of India, Bella Vista, Khairathabad Hyderabad, 2008 Vol-26, No.-3, P No. 297-303.

16. TaritKanti, Manzarul Hassan, ShafiualAzam, Jahingir Nagar University Dhaka,Bangladesh, Indian Journal of Public health, Jan 2008.

17. Veda Hedge, RD Kulkarni, GS Ajanta, Department of Oral Pathology, College of Dental Sciences, India, 2007, Vol. 11, Issue 1, P No.5 to 9

18. Abah\&Obimain, Journal of Public Health \& Epidemiology, Vol. 3, No. 3, March 2011, P No. 99-110.

19. Kabbash IA, EI Syed MM, Al Nawawy, AbouSaleem, Eastern Meditterranean Health Journal, 2007,13:392407. 